\title{
Adapting the range of validity for the Carleman linearization
}

\author{
Harry Weber and Wolfgang Mathis \\ Institute for Theoretical Electrical Engineering, Leibniz Universität Hannover, Hannover, Germany \\ Correspondence to: Harry Weber (weber@tet.uni-hannover.de)
}

Received: 15 January 2016 - Accepted: 11 March 2016 - Published: 28 September 2016

\begin{abstract}
In this contribution, the limitations of the Carleman linearization approach are presented and discussed. The Carleman linearization transforms an ordinary nonlinear differential equation into an infinite system of linear differential equations. In order to transform the nonlinear differential equation, orthogonal polynomials which represent solutions of a Sturm-Liouville problem are used as basis. The determination of the time derivate of this basis yields an infinite dimensional linear system that depends on the considered nonlinear differential equation. The infinite linear system has the same properties as the nonlinear differential equation such as limit cycles or chaotic behavior. In general, the infinite dimensional linear system cannot be solved. Therefore, the infinite dimensional linear system has to be approximated by a finite dimensional linear system. Due to limitation of dimension the solution of the finite dimensional linear system does not represent the global behavior of the nonlinear differential equation. In fact, the accuracy of the approximation depends on the considered nonlinear system and the initial value. The idea of this contribution is to adapt the range of validity for the Carleman linearization in order to increase the accuracy of the approximation for different ranges of initial values. Instead of truncating the infinite dimensional system after a certain order a Taylor series approach is used to approximate the behavior of the nonlinear differential equation about different equilibrium points. Thus, the adapted finite linear system describes the local behavior of the solution of the nonlinear differential equation.
\end{abstract}

\section{Introduction}

The large signal analysis of nonlinear circuits is an important subject in modern technologies of integrated circuits (Rugh, 1981). Most of the circuits are based on resistors, capacitors, inductors and semiconductor devices which are described by drift, diffusion and tunneling effects (Tsividis and Andrew, 2011). In the dynamical case such circuits are described by nonlinear differential equations. Solutions of these equations can only be obtained in special cases (Chua et al., 1987). Only in the case of linear differential equation with constant coefficients an analytic solution can be calculated in general.

The transformation of the nonlinear differential equation to linear differential equations are usually performed by a Taylor series in an operating point, which is truncated after the first order. By use of this technique the solution is only valid for small signals about the operating point.

Another linearization technique was developed by Carleman in 1932 (Carleman, 1932). He showed that polynomial differential equations can be represented by an infinite dimensional linear system. In 1981 Kerner showed that a vast of nonlinearities can be cast into an equivalent polynomial differential equation by increasing the dimension (Kerner, 1981). Therefore, most of the nonlinear circuits can be cast at first into a polynomial differential equation and further linearized by the Carleman linearization. Unfortunately, the infinite dimensional linear system cannot be solved in general. Therefore the infinite dimensional linear system has to be approximated by a finite dimensional linear system. This approach yields an approximation in the vicinity of the initial value and improves for initial values near to the origin.

In this contribution an adaption of the range of validity of the Carleman linearization is presented in order to describe the behavior of the system about different equilibrium points. For this purpose a short sketch of the Carleman technique is presented. In the following the adaption of the range of validity for the Carleman technique are explained and applied to some examples. 


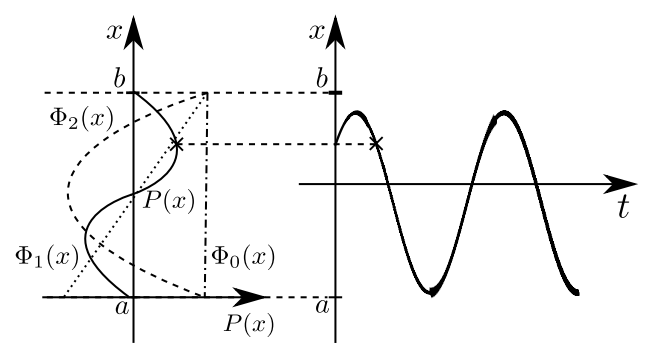

Figure 1. Representation of an arbitrary function $P(x)$ by orthogonal polynomials.

\section{Carleman linearization for ordinary differential equations}

The Carleman linearization provides a possibility to linearize polynomial differential equations

$$
\frac{\mathrm{d} x}{\mathrm{~d} t}=F(x)=\alpha_{0}+\alpha_{1} x+\alpha_{2} x^{2}+\cdots+\alpha_{p} x^{l}
$$

with initial value $x(0)$ (Kowalski and Steeb, 1991). In order to linearize Eq. (1) orthogonal polynomials $\Phi_{n}(x)$ of degree $n$ are used which represent solutions of the Sturm-Liouville equation (Lesky, 1962). By defining the scalar product

$$
(\Phi, \Psi):=\int_{\Omega} \Phi(x) \sqrt{w(x)} \Psi(x) \sqrt{w(x)} \mathrm{d} x
$$

on an interval $\Omega$ with the weighting function $w(x)$ orthogonal polynomials satisfy the condition

$\int_{\Omega} w(x) \Phi_{n}(x) \Phi_{m}(x) \mathrm{d} x=\delta_{n m}$

with

$\delta_{n m}=\left\{\begin{array}{lll}1 & \text { for } & n=m \\ 0 & \text { for } & n \neq m\end{array}\right.$

An arbitrary function $P(x)$ defined on $\Omega:=\operatorname{range}(x) \subset$ $\mathbb{R}$ can be described by a linear combination of orthogonal polynomials $\Phi_{n}(x)$ with $n=0,1,2, \cdots$ as shown in Fig. 1 for Jacobi polynomials in the interval $[a, b]$. Depending on $\Omega$ different polynomials can be used as complex orthogonal polynomials which are an equivalent complex representation of Hermite polynomials (Kowalski, 1997).

In order to transform Eq. (1) the differential equations for

$$
\Phi_{n}(x)=p_{n}(x)
$$

whereby $p_{n}(x)$ is defined as orthogonal polynomial are derived. For this purpose $\Phi_{n}(x)$ is differentiated

$$
\frac{\mathrm{d} \Phi_{n}(x)}{\mathrm{d} t}=\frac{\mathrm{d} x}{\mathrm{~d} t} \frac{\mathrm{d} \Phi_{n}(x)}{\mathrm{d} x}=F(x) \frac{\mathrm{d} \Phi_{n}(x)}{\mathrm{d} x}=G_{n}(x) .
$$

The new polynomial $G_{n}(x)$ is of degree $l+(n-1)$ due to the multiplication of the polynomial of degree $n-1$ and the vector field $F(x)$ of degree $l$. Representing $G_{n}(x)$ by a linear combination of orthogonal polynomials the infinite dimensional linear system $(n=0,1,2, \cdots)$

$\frac{\mathrm{d} \Phi_{n}(x)}{\mathrm{d} t}=G_{n}(x)=\sum_{i=0}^{\infty} a_{n, i} \Phi_{i}(x)$

with the initial values $\Phi_{n}(x(0))$ and

$a_{n, i}=\int_{\Omega} G_{n}(x) \Phi_{i}(x) w(x) \mathrm{d} x$

is obtained. The solution of Eq. (1) corresponds to $\Phi_{1}(x)$ since it represents a linear function in $x$. In the case of a linear $F(x)$ of degree $l \leq 1$ one obtains a finite dimensional linear system, for $l>1$ a coupled system of infinite linear differential equations is obtained. Due to simplicity the one dimensional case of Eq. (1) is presented. In the case of a $k$ dimensional polynomial differential equation in Eq. (1) the extension is achieved by

$\Phi_{\mathbf{n}}(\mathbf{x})=\prod_{i=1}^{k} p_{n_{i}}\left(x_{i}\right) \quad$ with $\quad \mathbf{n}=\left(n_{1}, n_{2}, n_{3}, \ldots\right)$.

\section{Approximation of the Carleman linearization}

With a few exceptions an infinite dimensional linear system Eq. (7) cannot be solved in general (see Kowalski and Steeb, 1991). Therefore the infinite dimensional linear system has to be approximated by a finite linear system. One way is to restrict the dimension to an arbitrary $N_{\max }<\infty$. In this case the infinite dimensional linear system Eq. (7) is approximated by $\left(n=0,1,2, \ldots, N_{\max }\right)$

$\frac{\mathrm{d} \Phi_{n}(x)}{\mathrm{d} t}=G_{n}(x) \approx \sum_{i=0}^{N_{\max }} a_{n, i} \Phi_{i}(x)$.

Each polynomial $G_{n}(x)$ of degree $l+(n-1)$ is approximated by a polynomial of maximal degree $N_{\max }$. If the degree of $G_{n}(x)$ is larger than $N_{\max }\left(l+(n-1)>N_{\max }\right)$ terms of higher degree are neglected. Therefore the truncated system provides only an approximation in the vicinity of the origin.

In order to describe the behavior of the system about different equilibrium points, a Taylor series of degree $N_{\max }$ is applied. Additionally to the limiting of the dimension to $N_{\max }$ each polynomial $G_{n}(x)$ which maximal degree is higher than $N_{\max }$ is approximated about an equilibrium point $x_{\mathrm{a}}$ of Eq. (1) by

$G_{n}(x) \approx T_{n}(x)=\left.\sum_{k=0}^{N_{\max }} \frac{\mathrm{d}^{(k)} G_{n}(x)}{\mathrm{d}^{(k)} x}\right|_{x=x_{\mathrm{a}}} \frac{1}{k !}\left(x-x_{\mathrm{a}}\right)^{k}$ 




Figure 2. Simple nonlinear dynamical circuit.

reducing the maximal degree to $N_{\max }$.

The resulting Taylor series $T_{n}(x)$ in Eq. (11) can be represented as a linear combination of orthogonal polynomials of maximal degree $N_{\max }$. In the next section the adapted method is applied to some examples and is compared to the truncated Carleman linearization.

\section{Carleman linearization and nonlinear circuits}

In order to illustrate the method of Sect. 2 a simple nonlinear circuit is considered, see Fig. 2.

The voltage $u$ is described by the differential equation

$\frac{\mathrm{d} u}{\mathrm{~d} t}=-\frac{I_{0}}{C}\left(1-\mathrm{e}^{u / u_{T}}\right)$

which can only be solved implicitly. In order to apply the Carleman linearization Eq. (12) has to be transformed into a polynomial differential equation. For this purpose the method proposed by Kerner is used by introducing the new variable

$i_{d}:=I_{0} \mathrm{e}^{u / u_{T}}$.

Using Eq. (13) Eq. (12) can be reformulated

$\frac{\mathrm{d} u}{\mathrm{~d} t}=-\frac{I_{0}}{C}+\frac{i_{d}}{C}$,

$\frac{\mathrm{d} i_{d}}{\mathrm{~d} t}=\frac{i_{d}}{u_{T}} \frac{\mathrm{d} u}{\mathrm{~d} t}=-\frac{I_{0}}{C u_{T}} i_{d}+\frac{i_{d}^{2}}{C u_{T}}$.

The polynomial in Eq. (15) corresponds to a quadratic polynomial differential equation of the form

$\frac{\mathrm{d} x}{\mathrm{~d} t}=\alpha_{1} x+\alpha_{2} x^{2}$

with real coefficients $\alpha_{1}$ and $\alpha_{2}$. In order to transform Eq. (16) into an infinite dimensional linear system the complex orthogonal polynomials $(n=0,1,2, \cdots)$

$\Phi_{n}(x)=\frac{x^{n}}{\sqrt{n !}}$

with $x: \mathbb{R} \rightarrow \mathbb{C}$ are used yielding the infinite dimensional linear system $(n=0,1,2, \cdots)$

$\frac{\mathrm{d} \Phi_{n}(x)}{\mathrm{d} t}=\alpha_{1} n \Phi_{n}(x)+\alpha_{2} n \sqrt{n+1} \Phi_{n+1}(x)$.

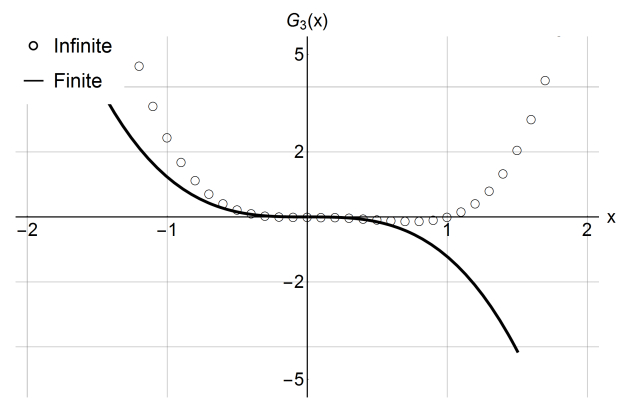

Figure 3. Comparison of $G_{3}(x)$ (circles) and truncated approximation in Eq. (19) (solid line) for Eq. (16) with $\alpha_{1}=-1$ and $\alpha_{2}=1$.

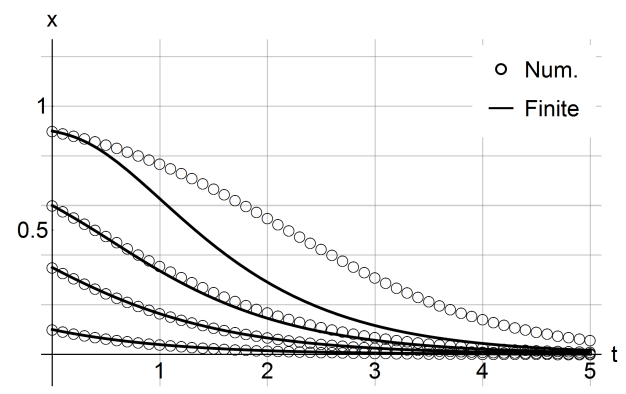

Figure 4. Comparison of the numerical solution (circles) and truncated Carleman linearization (solid line) for Eq. (16) with $\alpha_{1}=-1$ and $\alpha_{2}=1$.

In the following Eq. (18) is truncated after $N_{\max }=3$ resulting to the approximate polynomial

$$
\begin{aligned}
\frac{\mathrm{d} \Phi_{3}(x)}{\mathrm{d} t} & =G_{3}(x)=\alpha_{1} 3 \Phi_{3}(x)+\alpha_{2} 6 \Phi_{4}(x) \\
& \approx \alpha_{1} 3 \Phi_{3}(x) .
\end{aligned}
$$

In Fig. $3 G_{3}(x)$ is compared to the truncated approximation in Eq. (19) for the coefficients $\alpha_{1}=-1$ and $\alpha_{2}=1$. As one can see the finite system is only valid in the vicinity of $x=0$. The solution of the nonlinear differential equation are obtained from the truncated system by $\Phi_{1}(x)=x$. In Fig. 4 the numerical solution (circles) is compared to the truncated Carleman linearization (solid line) for different initial values. As shown, the error decreases for initial values in the vicinity of $x=0$ because the approximation of $G_{3}(x)$ is only valid about the origin.

In the following, another system is investigated which is described by

$\frac{\mathrm{d} x}{\mathrm{~d} t}=x-x^{2}$

and exhibits a stable equilibrium point at $x=1$. As shown in Eq. (19), $G_{3}(x)$ is approximated by a polynomial of degree three valid in the vicinity of the origin. In this example an adaption of the range of validity of the Carleman linearization about $x_{\mathrm{a}}=1$ is applied with $N_{\max }=3$ as shown 


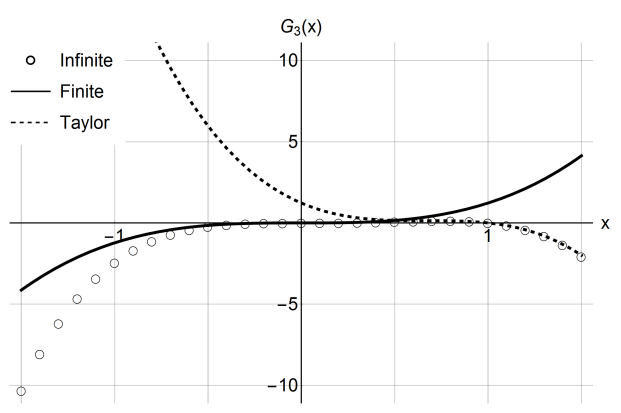

Figure 5. Comparison of $G_{3}(x)$ (circles), truncated system (solid line) and Taylor series approach (dashed line) for Eq. (20).

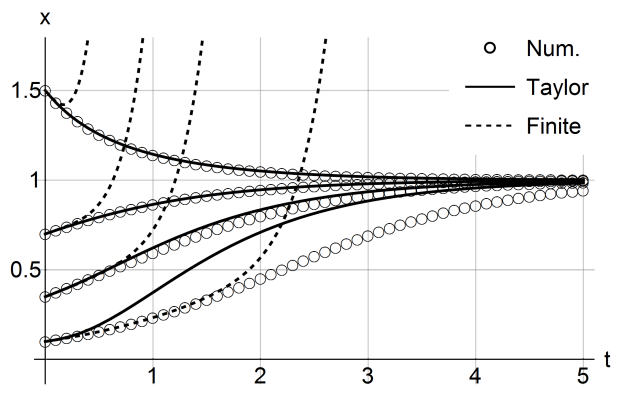

Figure 6. Comparison of the numerical solution (circles), truncated Carleman linearization (dotted line) and Taylor series approach (solid line) for Eq. (20).

in Eq. (11) for $n=3$. In Fig. 5 the function $G_{3}(x)$ is compared to the truncated Carleman linearization and the Taylor series approach. As shown the truncated function is only valid in the vicinity of $x=0$, whereas the Taylor series approach are valid about $x=1$. After solving the truncated and the adapted system the solution of Eq. (20) are obtained by $\Phi_{1}(x)=x$. In Fig. 6 solutions of both approaches are compared to the numerical results of Eq. (16). As shown the Taylor series approach approximates the steady state behavior of the differential equation whereas the truncated approach is only valid for a short time period in the vicinity of the initial value. Further it is shown that for initial values not in the vicinity of $x=1$ the error of the Taylor series approach increases since the region of validity is left as shown in Fig. 5.

Now the numerical solution of the differential equation

$\frac{\mathrm{d} x}{\mathrm{~d} t}=4 x-x^{3}$

is compared to the Taylor series approach about the equilibrium point $x_{\mathrm{a}}=2$ for different $N_{\max }$. In Fig. 7 the comparison of both solutions is presented for $N_{\max }=3$ and $N_{\max }=8$. As shown the error of the approximation decreases with increased order but this is only valid for initial values in the vicinity of the equilibrium point $x_{\mathrm{a}}$ since only a local approximation is possible with the Taylor series. Therefore this approach cannot be used to describe the behavior of differential equations for a larger range of initial values.

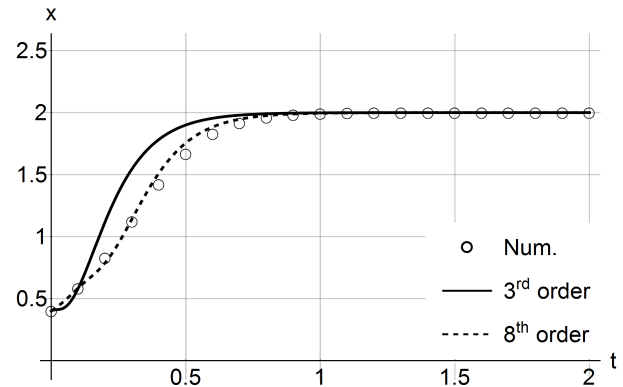

Figure 7. Comparison of the numerical results (circles), Taylor series approach of 3rd (solid line) and 8th (dotted line) order for Eq. (21).

\section{Conclusions}

In this contribution the Carleman linearization is presented which transforms a nonlinear differential equation into an infinite dimensional linear system. Unfortunately, the infinite dimensional system cannot be solved in general. Therefore the system is truncated after an arbitrary order yielding an approximation about the initial value for a short time period. In order to describe the asymptotic behavior about different equilibrium points a Taylor series approach was presented which is limited to the operating point of the series. In a forthcoming paper a self-consistent technique will be discussed in order to represent the behavior of the polynomial differential equation on an interval.

The publication of this article was funded by the open-access fund of Leibniz Universität Hannover.

Edited by: J. Anders

Reviewed by: two anonymous referees

\section{References}

Carleman, T.: Application de la théorie des équations intégrales linéaires aux systèmes d'équations différentielles non linéaires, Acta Mathematica, 1932.

Chua, L. O., Desoer, C. A., and Kuh, E. S.: Linear and Nonlinear Circuits, McGraw-Hill Book Company, 1987.

Kerner, E. H.: Universal formats for nonlinear ordinary differential systems, J. Math. Phys., 22, 1366-1371, 1981.

Kowalski, K.: Nonlinear dynamical systems and classical orthogonal polynomials, J. Math. Phys., 38, 2483-2505, 1997.

Kowalski, K. and Steeb, W.-H.: Nonlinear Dynamical Systems and Carleman Linearization, World Scientific, 1991.

Lesky, P.: Die charakterisierung der klassischen orthogonalen polynome durch Sturm-Liouvillesche Differentialgleichungen, Archive for Rational Mechanics and Analysis, 10, 341-351, 1962.

Rugh, W. J.: Nonlinear System Theory, The Johns Hopkins University Press, 1981.

Tsividis, Y. and Andrew, C. M.: Operation and Modeling of the MOS Transistor, OXFORD University Press, 2011. 